\title{
Interventions for smoking cessation in hospitalised patients: a systematic review
}

\author{
M Munafò, N Rigotti, T Lancaster, L Stead, M Murphy
}

\begin{abstract}
Background-An admission to hospital provides an opportunity to help people stop smoking. Individuals may be more open to help at a time of perceived vulnerability, and may find it easier to quit in an environment where smoking is restricted or prohibited. Providing smoking cessation services during hospitalisation may help more people to attempt and sustain an attempt to quit. The purpose of this paper is to systematically review the effectiveness of interventions for smoking cessation in hospitalised patients.

Methods-We searched the Cochrane Tobacco Addiction Group register, CINAHL, and the Smoking and Health database for studies of interventions for smoking cessation in hospitalised patients. Randomised and quasi-randomised trials of behavioural, pharmacological, or multicomponent interventions to help patients stop smoking conducted with hospitalised patients who were current smokers or recent quitters were included. Studies of patients admitted for psychiatric disorders or substance abuse, those that did not report abstinence rates, and those with follow up of less than 6 months were excluded. Two of the authors extracted data independently for each paper, with assistance from others.
\end{abstract}

Results-Intensive intervention (inpatient contact plus follow up for at least 1 month) was associated with a significantly higher cessation rate compared with controls (Peto odds ratio (OR) $1.82,95 \%$ CI 1.49 to 2.22). Any contact during hospitalisation followed by minimal follow up failed to detect a statistically significant effect on cessation rate, but did not rule out a $30 \%$ increase in smoking cessation (Peto OR $1.09,95 \%$ CI 0.91 to 1.31 ). There was insufficient evidence to judge the effect of interventions delivered only during the hospital stay. Although the interventions increased quit rates irrespective of whether nicotine replacement therapy (NRT) was used, the results for NRT were compatible with other data indicating that it increases quit rates. There was no strong evidence that clinical diagnosis affected the likelihood of quitting.
Conclusions-High intensity behavioural interventions that include at least 1 month of follow up contact are effective in promoting smoking cessation in hospitalised patients.

(Thorax 2001;56:656-663)

Keywords: smoking cessation; inpatients

A hospital stay may provide a good setting in which to deliver smoking cessation intervention, and hospitalisation may boost receptivity to smoking cessation messages by increasing perceived vulnerability to the health hazards of tobacco use. This effect may be strongest with tobacco related illnesses if the presence of such an illness increases the extent, for example, with which an intervention is complied. This may be as a result of the extent to which patients perceive smoking as a cause of their disease, thereby influencing the perceived gain associated with cessation. Many hospitals are smoke free, which may provide a supportive environment within which to begin a cessation attempt by providing an opportunity to abstain away from the usual cues to smoke. Illness also brings smokers into contact with health professionals who can provide a smoking cessation message or intervention. Procedures such as coronary arteriography that provides detail of the patient's cardiac status may minimise the subsequent denial of cardiac risk by the patient. ${ }^{12}$ For these reasons, tobacco dependence interventions delivered (or initiated) in hospitals might be particularly effective.

Before investing in such services the evidence regarding their efficacy needs to be reviewed systematically. The efficacy of hospital based intervention may differ according to the type and intensity of the intervention method and the nature of the patient's illness. Interventions can provide behavioural counselling, pharmacotherapy, or both, varying in intensity and duration.

The primary aim of this review is to evaluate the effectiveness of smoking cessation interventions directed at the hospitalised patient. In order to guide policy, we also aim to identify the components of effective programmes and to explore whether there is a difference in effect according to the patient's reason for hospitalisation. 
Our hypotheses were that:

- Systematic behavioural intervention (brief advice, individual counselling, provision of self-help materials, group therapy) increases quit rates more than usual care, and that intensive intervention increases quit rates more than brief intervention.

- Interventions that occur both in hospital and after discharge increase quit rates more than interventions limited to the hospital stay, and that longer post-discharge follow up increases quit rates more than short follow up.

- Adding nicotine replacement therapy (NRT) to a behavioural intervention increases quit rates more than placebo or no medication, and that combining NRT and a behavioural intervention increase quit rates more than either alone.

A secondary objective was to explore the possibility that the efficacy of interventions differs for patients with different diagnoses. This was done using subgroup analyses by disease category. These analyses were post hoc and exploratory in nature, and designed only to generate hypotheses.

This review is a shortened version of a systematic review undertaken for the Cochrane Collaboration Tobacco Addiction review group. ${ }^{3}$ The Cochrane review will be regularly updated to take account of new research.

\section{Methods}

SELECTION OF STUDIES FOR INCLUSION

Types of study

We included randomised or quasi-randomised controlled trials that recruited patients who were hospitalised or about to be hospitalised and who were currently smoking or had recently quit. We excluded trials of secondary prevention or cardiac rehabilitation that did not recruit on the basis of smoking history and trials on patients hospitalised for psychiatric disorders or substance abuse (including inpatient tobacco addiction programmes). Trials in which "recent quitters" were classified as smokers were included, but a sensitivity analysis was performed on these data to determine whether they differed from trials that excluded such individuals.

\section{Types of interventions}

We included any intervention to increase smokers' motivation to quit, to assist them in making a quit attempt or, in the case of recent quitters, to help them avoid relapse. The intervention could be delivered by physicians, nursing staff, psychologists, smoking cessation counsellors, or other hospital staff. The intervention could include advice or more intensive behavioural therapy, with or without the use of pharmacotherapy or post-discharge follow up. The control intervention could be usual care or any less intensive programme such as brief advice only. We included studies of smoking interventions that were part of a broader rehabilitation programme only if it was possible to extract data on the outcome effects of the smoking cessation component specifically, and if details of the nature of the intervention and control were explicitly stated. Studies that reported the use of NRT or other pharmacotherapy were included.

Interventions during the hospital stay were categorised according to whether they included follow up after discharge. We categorised interventions by the following levels of intensity: (1) Single contact in hospital lasting $\leqslant 15$ minutes, no follow up support.

(2) One or more contacts in hospital lasting in total $>15$ minutes, no follow up support.

(3) Any hospital contact plus follow up of $\leqslant 1$ month.

(4) Any hospital contact plus follow up $>1$ month.

\section{Types of outcome measures}

The principal outcome measure was abstinence from smoking at least 6 months after the start of the intervention. We used the most conservative measure of quitting at the longest follow up-that is, a biochemically validated sustained quit rate was used in preference to self-reported point prevalence abstinence, and abstinence at 12 month follow up was used in preference to abstinence at 6 month follow up. We counted participants lost to follow up as continuing smokers.

SEARCH STRATEGY

We searched the Tobacco Addiction Group trials register to July 2000. This specialised register is regularly updated by electronic searches and hand searching. Searches for the register covered smoking cessation, nicotine dependence, nicotine addiction, and tobacco use. In addition, we searched the Cochrane Controlled Trials Register, the Centers for Disease Control Smoking and Health database, and CINAHL. Individuals with expertise in the area of smoking cessation were asked for details of conference abstracts and studies in press. Bibliographies of studies generated by the search were hand checked for further studies.

Search strategy for Tobacco Addiction specialised register and for CCTR (used in combination with the terms specific to tobacco used to identify records for specialised register): (hospital and patient ${ }^{\star}$ ) or hospitali ${ }^{\star}$ or inpatient ${ }^{\star}$ or admission ${ }^{\star}$ or admitted

Search strategy for Smoking and Health database (National Center for Chronic Disease Prevention and Health Promotion CDP File). For records with descriptor indexing: (SMOKING-CESSATION ${ }^{\star}$ in DE) AND ((hospital ${ }^{\star}$ AND patient ${ }^{\star}$ ) OR inpatient ${ }^{\star}$ OR admission $\left.{ }^{\star}\right)$. For records without descriptor indexing: (smoking near (cessation OR quit* OR stop $\left.{ }^{\star}\right)$ AND ((HOSPITAL ${ }^{\star}$ AND patient $\left.^{\star}\right)$ OR inpatient ${ }^{\star}$ OR admission $\left.{ }^{\star}\right)$.

Search strategy for CINAHL (Silverplatter): 1 ((hospital with patient ${ }^{\star}$ ) in TI OR AB 2 (hospitali ${ }^{\star}$ OR inpatient ${ }^{\star}$ OR admission* OR admitted) in TI OR AB

3 (hospitali* OR inpatient ${ }^{\star}$ ) in DE

4 (quit $^{\star}$ OR smok ${ }^{\star}$ OR cigar ${ }^{\star}$ OR tobacco OR nicotine) in TI OR AB

5 (smok ${ }^{\star}$ OR tobacco OR nicotine) in DE (1 OR 2 OR 3) AND (4 OR 5) 
$\mid$

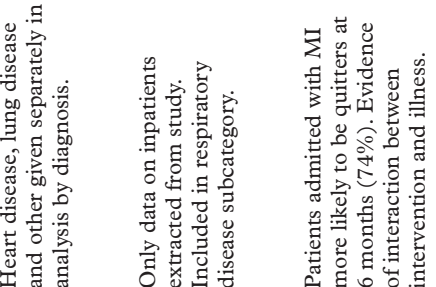

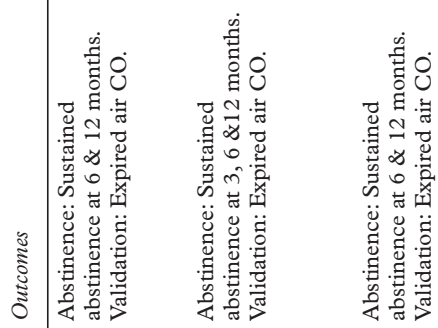

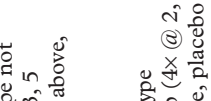

क्ष

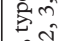

可

宫高

矛遂

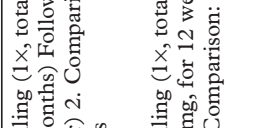

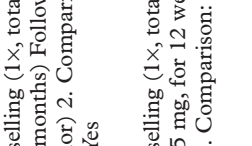

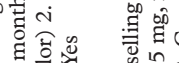

年

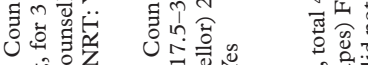

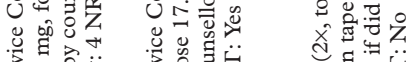

क्ष

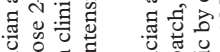

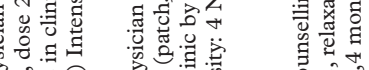

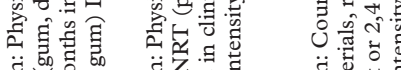

蒡

政

竎

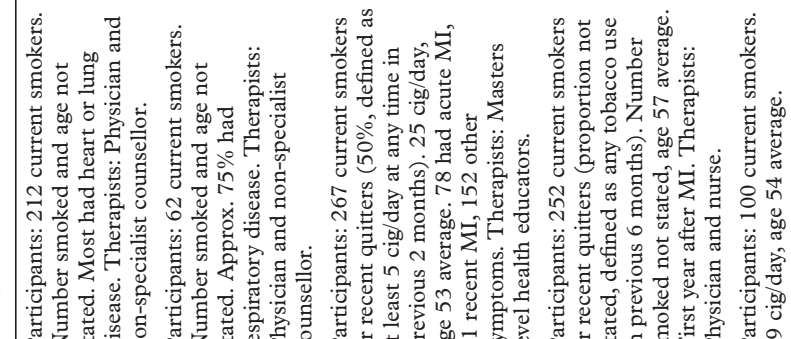

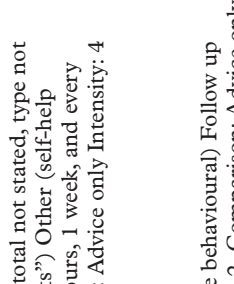

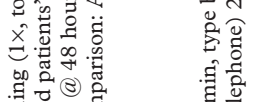

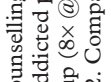

ن.

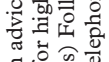

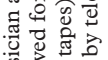

蛋悹

范行

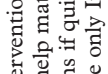

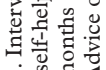

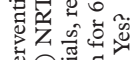

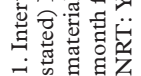

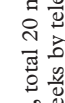
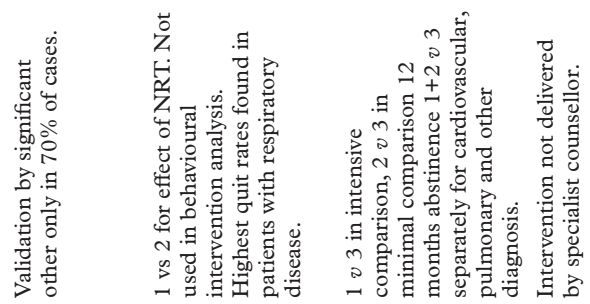

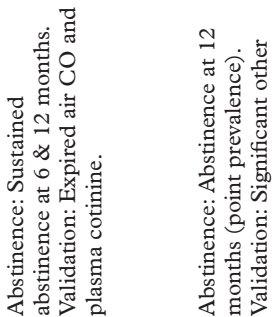

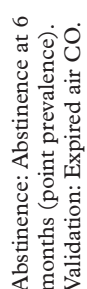

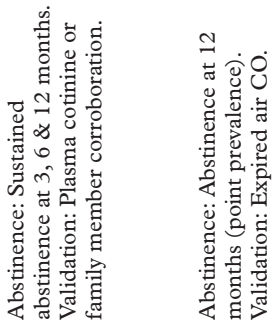

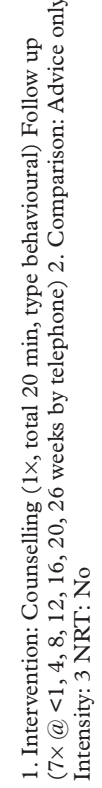

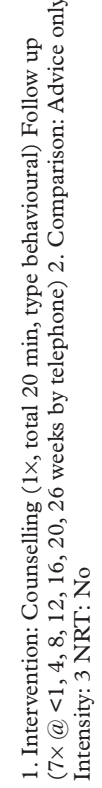

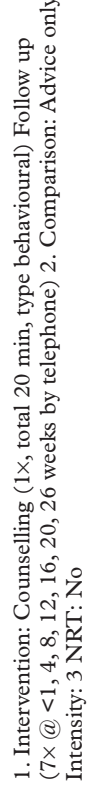

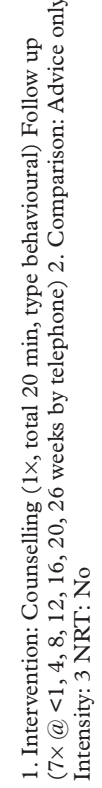

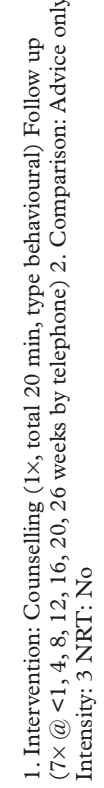

4

र्ष

这昆离

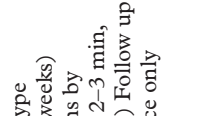

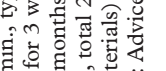

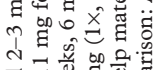

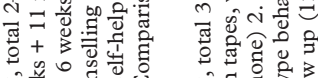

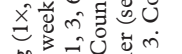

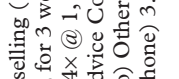

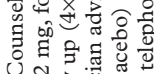

O

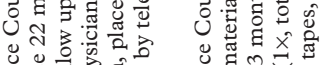

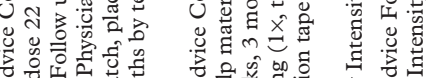

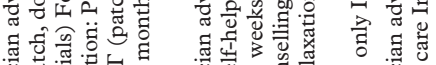

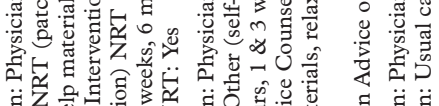

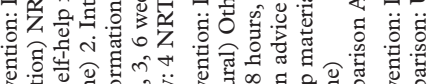

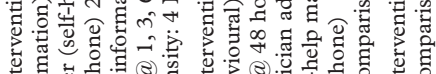

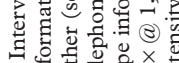

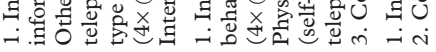

\section{总}

苞

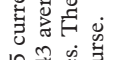

象舟,

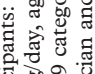

혈

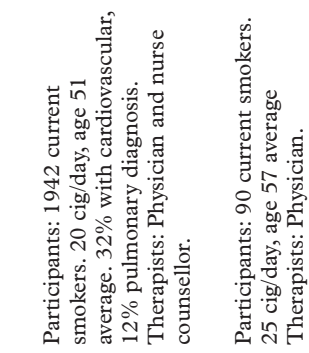

:

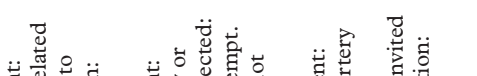

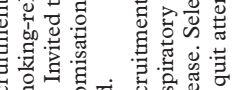

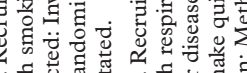

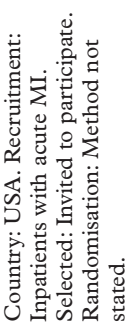

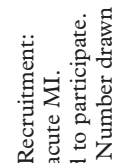

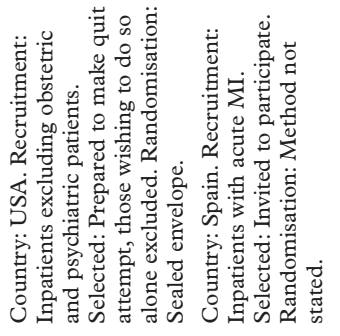

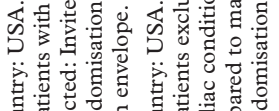

WWII

新

竞

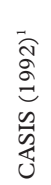

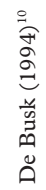

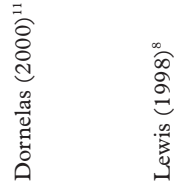

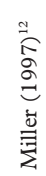

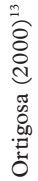

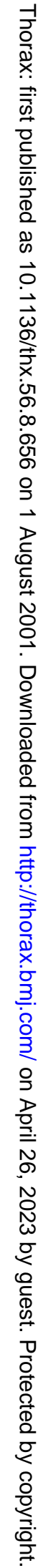




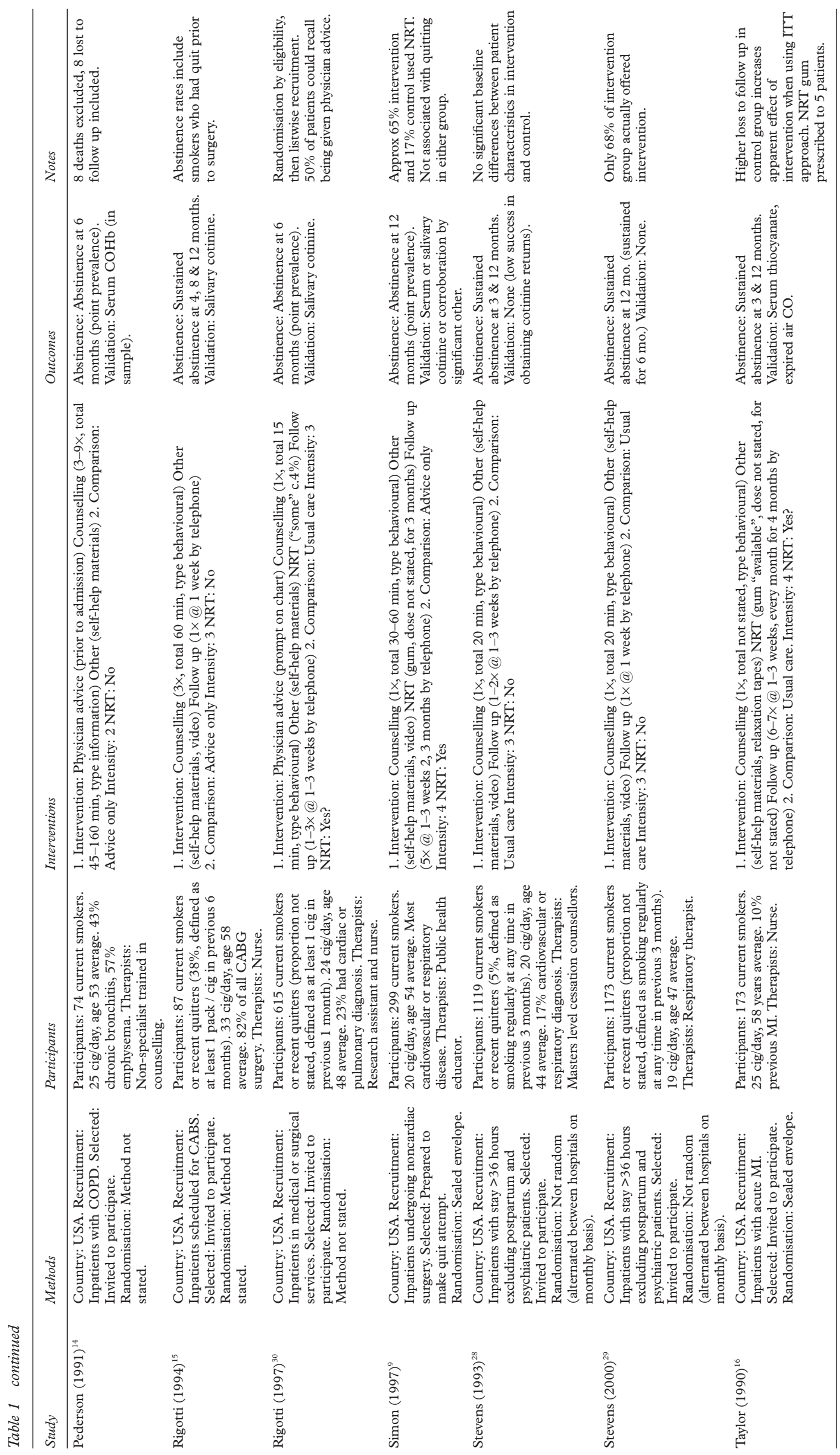


Table 2 Characteristics of excluded studies

\begin{tabular}{ll}
\hline Study & Reason for exclusion \\
\hline Allen $(1998)^{17}$ & Not inpatients (delivered at outpatient clinic) \\
BTS $(1983)^{18}$ & Inpatient and outpatient data not reported separately \\
Burt $(1974)^{19}$ & Not randomised \\
Colby $(1998)^{20}$ & Short follow up (3 months). Only investigated adolescent smokers \\
Dale (1995) & Not inpatients (some participants admitted to inpatients unit for smoking \\
& intervention) \\
Gritz $(1993)^{22}$ & Not inpatients (only recruitment carried out in hospital setting) \\
Johnson $(1999)^{23}$ & Not randomised \\
Meenan $(1998)^{24}$ & Not randomised \\
Schmitz $(1999)^{25}$ & No control/usual care group \\
Strecher $(1985)^{26}$ & Not randomised \\
Wewers $(1994)^{27}$ & Short follow up (5 weeks) \\
\hline
\end{tabular}

EXTRACTION OF CITATIONS

Three authors checked studies identified by the search strategies for relevance. One author extracted data independently with checking by a second. Disagreements were resolved by mutual consent. Reasons for the exclusion of studies were noted. For each study we extracted the following data:

(1) author(s) and year of publication,

(2) methods (country of origin, recruitment, randomisation and participants),

(3) description of intervention(s) and control, including a designation of intensity (1-4), and

(4) outcomes (length of follow up, definition of abstinence, validation technique).

If necessary, the original authors were contacted for clarification of data. Studies were evaluated on the basis of the quality of the randomisation procedure used, as this is the source of bias which has been empirically associated with overestimation of treatment effects. ${ }^{4}$ We also assessed whether the studies reported validation of self-reported smoking cessation, how they handled patients lost to follow up, and the extent to which populations consisted of current smokers and recent quitters since these are possible sources of bias.

\section{ANALYSIS OF DATA}

The statistical methods used for pooling were as described by Peto's group. ${ }^{5}$ The results are expressed as the Peto odds ratio (POR) (intervention/control) for achieving abstinence from smoking together with the $95 \%$ confidence interval for this estimate. Tests for heterogeneity were performed using a MantelHaenszel $\chi^{2}$ statistic.

Quit rates were calculated based on the numbers of patients randomised to an intervention, excluding any deaths. Those who dropped out or were lost to follow up were counted as continuing smokers.

Effectiveness was evaluated according to our predetermined classification of four levels of intensity. Where we included studies that were judged by quality criteria to be more prone to bias, we planned sensitivity analyses to assess whether their inclusion altered our findings. Sensitivity analyses were also planned to explore, where possible, the contribution of different components to any overall effect (for example, the role of NRT in a multicomponent intervention) and to determine whether the effects were different when the study population was restricted to those wishing to stop.
In an exploratory analysis we evaluated effectiveness in patients with a diagnosis of cardiovascular disease, respiratory disease, and cancer. In cases where a single study reported data on patients from different categories we pooled the data only when it was possible to extract data by disease category. Otherwise, we included only those studies reporting data from patients in a single disease category.

\section{Results}

DESCRIPTION OF STUDIES

Fifteen trials conducted in the USA, UK, and Spain between 1990 and 2000 met the inclusion criteria and contributed to the review. The characteristics of the studies are described in table 1.

All but two of these studies contributed to the main comparison of intensity of intervention versus control. Those that did not contribute $^{67}$ did not include a usual care control group. Seven studies included NRT as a component, of which four studies ${ }^{6-9}$ used NRT as a specific component offered to all participants receiving the intervention as opposed to only a subgroup. Ten studies ${ }^{16710-16}$ provided separate data by disease and contributed to the exploratory comparison of intervention in different disease categories versus control. We excluded 11 studies $^{17-27}$ which appeared relevant but did not meet all inclusion criteria. These are described in table 2.

\section{QUALITY OF STUDIES}

Five of the 15 studies reported an adequate randomisation procedure. ${ }^{89111216}$ Two studies allocated treatment by alternating between hospitals over time. ${ }^{28} 29$ The remaining eight studies did not report the method of randomisation.

\section{META-ANALYSIS}

Hospital interventions categorised by intensity

No included studies reported on the effects of brief interventions in hospitalised patients (intensity 1 ). One study ${ }^{14}$ reported that a more intensive intervention in hospital but with no follow up after discharge (intensity 2) increased quit rates (POR 1.64, 95\% CI 0.54 to 5.02 ). There is thus limited evidence available to determine whether intervention confined to the hospital stay increases quit rates. Analysis of six studies reporting on the effects of any contact during hospitalisation followed by minimal follow up (intensity 3) failed to detect a statistically significant effect, but did not rule out a 30\% increase in smoking cessation (POR $1.09,95 \%$ CI 0.91 to 1.31 ). Pooled analysis of seven studies reporting on the effects of more intensive follow up following an intervention in hospital (intensity 4) shows a statistically significant increase in quit rates (POR 1.82, $95 \%$ CI 1.49 to 2.22 ; fig 1 ).

Sensitivity analysis: A sensitivity analysis excluding four studies that reported the use of NRT within the highest intervention intensity ${ }^{8-}$ 1016 did not suggest that the efficacy of these interventions was due to the use of NRT (POR $1.61,95 \%$ CI 1.26 to 2.06 ). Only one study that delivered a minimal intensity intervention 


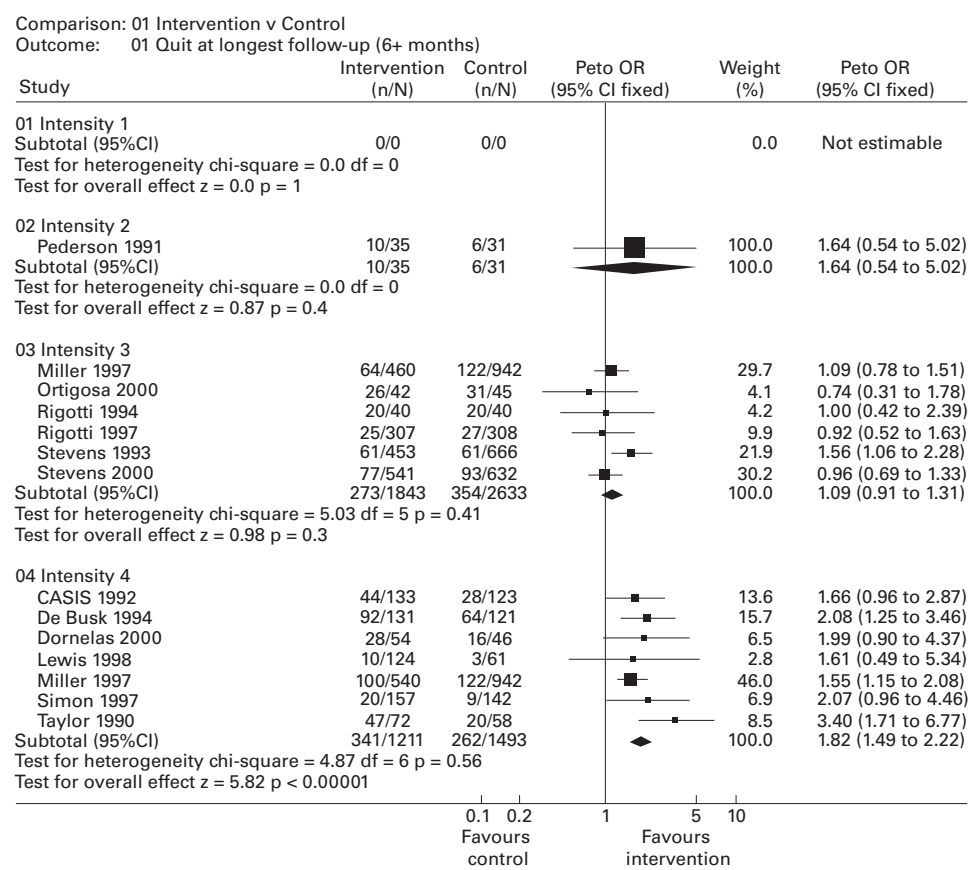

Figure 1 Effects of intervention by intensity.

with follow up (intensity 3) reported the use of $\mathrm{NRT}^{30}$ but this was in only about $4 \%$ of participants so a sensitivity analysis was not possible. Within studies that delivered an intervention with minimal follow up (intensity

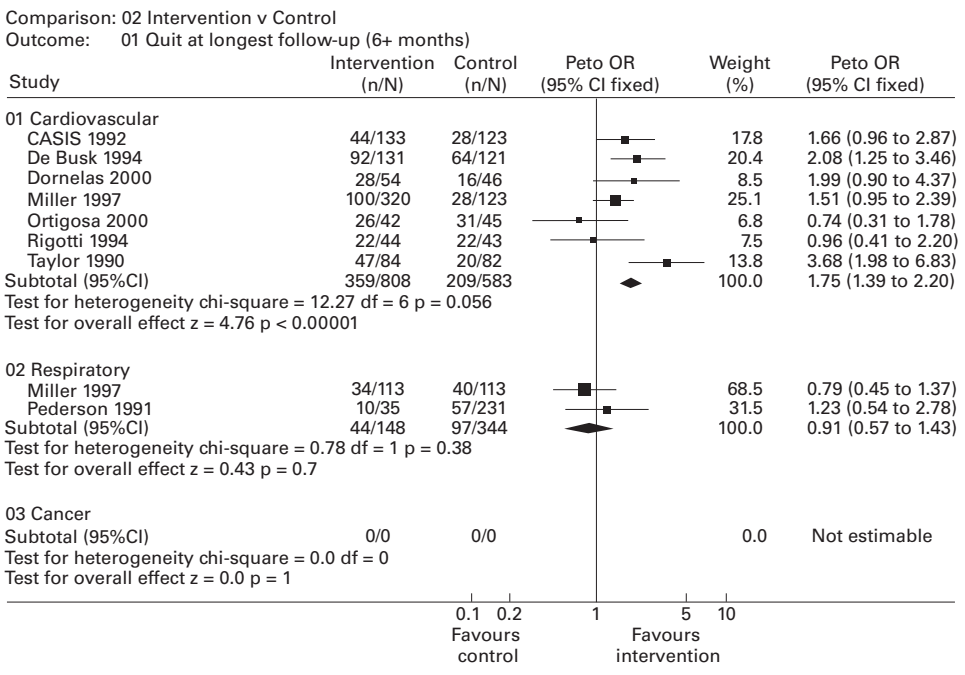

Figure 2 Effects of intervention by diagnosis.

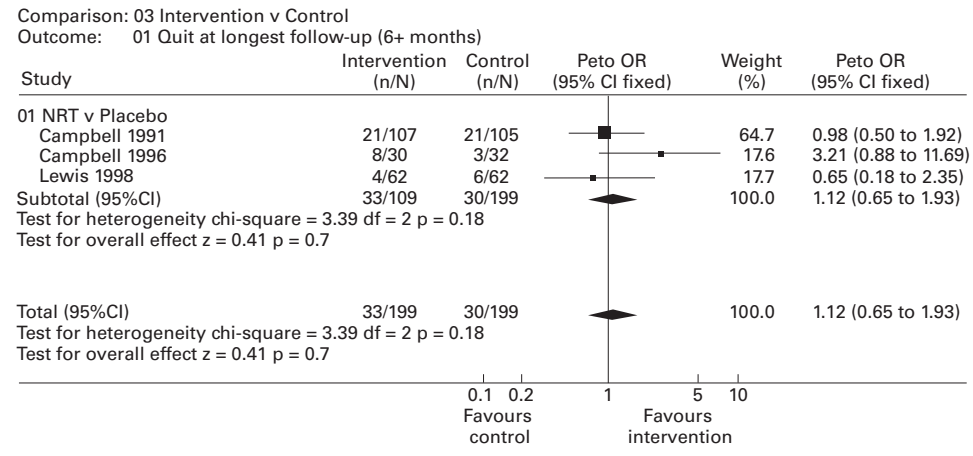

Figure 3 Effects of nicotine replacement therapy.
3) we performed a sensitivity analysis excluding the data from two studies that did not randomise patients. ${ }^{28}{ }^{29}$ This changed the point estimate but did not significantly affect the confidence intervals (POR 1.01, 95\% CI 0.78 to 1.31). Within studies that delivered the highest intervention intensity (intensity 4 ) we performed a sensitivity analysis excluding the data reported by studies in which participants were selected on the basis of willingness to make a quit attempt. ${ }^{812}$ There continued to be an effect on quit rates in the remaining studies (POR 2.12, 95\% CI 1.57 to 2.87 ). We performed a sensitivity analysis excluding studies that reported data from recent quitters as well as current smokers. ${ }^{1}{ }^{10} 152830$ In the case of studies delivering a minimal intensity intervention with follow up (intensity 3) there was little change in the estimates (POR 1.04, 95\% CI 0.76 to 1.41$)$. In studies delivering the highest intervention intensity (intensity 4 ) the increase in quitting remained significant (POR $1.79,95 \%$ CI 1.41 to 2.28 ). Finally, we performed a sensitivity analysis excluding studies that reported point prevalence cessation data rather than sustained abstinence data. $^{8911} 131430$ This excluded the only study reporting on the effects of brief intervention alone (intensity 1 ). In the case of intervention with minimal follow up (intensity 3 ) the increase in quitting remained non-significant (POR $1.14,95 \%$ CI 0.94 to 1.38 ), while in the case of the highest intervention intensity (intensity 4) the increase in quitting remained significant (POR 1.79, 95\% CI 1.44 to 2.23 ).

\section{Effect of intervention by diagnosis}

Seven studies ${ }^{10-13} 1516$ reported on the effects of interventions in patients hospitalised with a cardiovascular diagnosis (fig 2). Pooled analysis of these studies suggested that intervention increased quitting (POR $1.75,95 \%$ CI 1.39 to 2.20). Two studies ${ }^{12}{ }^{14}$ reported on interventions in patients hospitalised with a respiratory diagnosis. Pooled analysis failed to detect an increase in cessation rates compared with the control group (POR 0.91, 95\% CI 0.57 to 1.43) but the confidence interval does not rule out a clinically useful effect. No study reported on the effects of interventions in patients hospitalised with a diagnosis of cancer.

Sensitivity analysis: We performed a sensitivity analysis excluding studies that reported point prevalence cessation data rather than sustained abstinence data. ${ }^{11} 1314$ In patients hospitalised with a cardiovascular diagnosis this led to a slight increase in the likelihood of quitting (POR $1.85,95 \%$ CI 1.44 to 2.37 ). In patients admitted with a respiratory diagnosis the effects of intervention remained nonsignificant (POR $0.79,95 \%$ CI 0.45 to 1.37 ).

\section{Effects of NRT}

Analysis of three studies ${ }^{6-8}$ comparing the use of NRT with placebo NRT failed to detect a statistically significant effect (fig 3). However, the confidence interval was wide and does not rule out the possibility of a more than $50 \%$ increase in smoking cessation due to the use of 
NRT as part of an inpatient smoking cessation intervention (POR 1.12, 95\% CI 0.65 to 1.93 ).

Sensitivity analysis: We performed a sensitivity analysis excluding one study that reported point prevalence cessation data rather than sustained abstinence data. ${ }^{8}$ The effects of intervention remained non-significant (POR $1.2695 \%$ CI 0.69 to 2.29 ).

We detected no significant heterogeneity in any of the analyses.

\section{Discussion}

The results of this review suggest that smoking cessation interventions delivered during a period of hospitalisation, with follow up support after discharge, increase smoking cessation. There are insufficient studies to determine the effects of interventions delivered only during hospitalisation. However, there is evidence from other populations that brief advice is an effective intervention in promoting smoking cessation. ${ }^{31}$ There is also evidence from other sources that smoking behaviour early in the inpatient cessation attempt is a strong predictor of long term remission. ${ }^{32}$ There is indirect evidence that increasing intensity of intervention, particularly by increasing the amount of follow up contact after discharge, is associated with higher rates of cessation. This suggests that post-discharge follow up may be an important part of interventions delivered initially during the hospitalisation period. Unfortunately, one of the largest studies carried out on hospitalised patients $^{18}$ was excluded because it did not report separate data for inpatient and outpatient groups.

It is not possible to determine how much NRT contributes to the effect of hospital interventions as all studies that used this were also categorised as high intensity on the basis of the degree of post-discharge follow up. Exclusion of those studies that reported the use of NRT did not reduce the apparent efficacy of these interventions. An analysis of studies that compared the use of NRT with placebo NRT delivered within a high intensity intervention also failed to detect a statistically significant effect. However, the confidence interval was compatible with an effect of NRT similar to that found in other settings. ${ }^{33}$ Similarly, while no study considered the use of antidepressants in hospitalised patients, there is increasing evidence from other populations that they help to sustain a quit attempt ${ }^{34}$ and can be considered in such patients where there is no clinical contraindication.

In the case of the highest intensity interventions (intensity 4), neither the exclusion of studies that included recent quitters as well as current smokers nor those that included patients selected for motivation significantly affected the quit rates achieved. Treatments of nicotine dependence should be matched to the needs of the individual, but these data support the idea that help of some form be offered to all smoking patients, irrespective of motivation to quit.

The analyses by diagnosis suggest that there is an increase in cessation rate in patients with a cardiovascular diagnosis given an intervention. As these were not patients selected by motivation to quit, this finding lends some support to the hypothesis that patients with a smoking related illness are more receptive to intervention. The effectiveness of interventions in patients with a respiratory diagnosis is less clear, generating the hypothesis that patients with some diagnoses may find it more difficult to quit. However, the confidence intervals were wide and overlap with the estimates in patients with cardiovascular disease. Hence, there is no strong evidence for a differential effect by diagnosis. Although different diagnoses may provide greater or lesser motivation to make a quit attempt, the degree of tobacco dependence is likely to be a stronger indicator of ability to quit than clinical diagnosis.

The results support the use of smoking cessation interventions delivered during the hospitalisation period that also include follow up for at least 1 month after discharge. Although such interventions were effective whether or not NRT was used, the results are compatible with data which show the effectiveness of NRT in other populations. There was no clear evidence that patients with different clinical diagnoses respond in different ways.

There are some notable areas where there is little or no research available that is eligible for inclusion in this review. In particular, there are no studies that report on the efficacy of brief advice during the inpatient period with no subsequent follow up, and only one study that reported on the efficacy of intensive advice delivered during the inpatient period with no subsequent follow up.

It was also not possible to examine the impact of interventions delivered to patients with a diagnosis of cancer. Although several studies reported data on interventions delivered to patients with a cardiovascular or respiratory diagnosis, the lack of evidence with respect to patients with cancer suggests another area where research is required.

The authors would like to thank Sarah Welch and Sarah Roberts of the ICRF General Practice Research Group for their assistance in extracting data. Ian Campbell, Chris Silagy and Corinne Husten provided helpful comments on earlier drafts. Conflict of interest: One of the authors (Rigotti) was an author of two of the studies included in the review. ${ }^{15} 30$

1 Ockene JK, Kristeller J, Goldberg R, et al. Smoking cessation and severity of disease: The Coronary Artery Smoking and severity of disease: The Coronary Artery Sm

2 Rosal MC, Ockene JK, Ma YS, et al. Coronary Artery Smoking Intervention Study (CASIS): 5-year follow up.
Smatery Health Psychol 1998;17:476-8.

3 Rigotti NA, Munafò M, Stead LF, et al. Interventions for smoking cessation in hospitalised patients. In: Cochrane Library, Issue 1, 2001. Oxford: Update Software.

4 Schulz KF, Chalmers I, Hayes RJ, et al. Empirical evidence of bias: dimensions of methodological quality associated with treatment effects in controlled trials. $\mathscr{F} A M A$ 1995;273: 408-12.

5 Yusuf S, Peto R, Lewis J, et al. Beta blockade during and after myocardial infarction: an overview of the randomized trials. Prog Cardiovasc Dis 1985;27:335-71.

6 Campbell IA, Prescott RJ, Tjeder-Burton SM. Smoking cessation in hospital patients given repeated advice plus nicotine or placebo chewing gum. Respir Med 1991;85: 155-7.

7 Campbell IA, Prescott RJ, Tjeder-Burton SM. Transdermal nicotine plus support in patients attending hospital with smoking-related diseases: a placebo controlled study. Respir
Med 1996;90:47-51. 
8 Lewis SF, Piasecki TM, Fiore MC, et al. Transdermal nicotine replacement for hospitalized patients: a randomized tine replacement for hospitalized patien
clinical trial. Prev Med 1998;27:296-303.

9 Simon JA, Solkowitz SN, Carmody TP, et al. Smoking cessation after surgery: a randomized trial. Arch Intern Med 1997;157:1371-6.

10 DeBusk RF, Houston-Miller N, Superko HR, et al. A casemanagement system for coronary risk factor modification after acute myocardial infarction. Ann Intern Med 1994 120:721-9.

11 Dornelas EA, Sampson RA, Gray JF, et al. A randomised controlled trial of smoking cessation counseling after myocardial infarction. Prev Med 2000;30:261-8.

12 Miller NH, Smith PM, DeBusk RF, et al. Smoking cessation in hospitalized patients: results of a randomized trial. Arch Intern Med 1997;157:409-15.

13 Ortigosa AM, Gomez FJO, Ramalle-Gomara E, et al. Eficacia de una intervencion para dejar de fumar en pacientes con infarto de miocardio [Efficacy of an intervention in smoking cessation in patients with myocardial infarction]. Med Clin (Barc) 2000;114:209-10.

14 Pederson LL, Wanklin JM, Lefcoe NM. The effects of counseling on smoking cessation among patients hospitalized with chronic obstructive pulmonary disease: randomized clinical trial. Int $\mathcal{F}$ Addict 1991;26:107-19.

15 Rigotti NA, McKool KM, Shiffman S. Predictors of smoking cessation after coronary artery bypass graft surgery: results of a randomized trial with 5-year follow-up. Ann Intern Med 1994;120:289-93.

16 Taylor CB, Houston-Miller NH, Killeen JD, et al. Smoking cessation after acute myocardial infarction: effects of a nurse-managed intervention. Ann Intern Med 1990;113 118-23.

17 Allen B, Pederson LL, Leonard EH. Effectiveness of physicians-in-training counseling for smoking cessation in African Americans. F Nat Med Assoc 1998;90:597-604.

18 British Thoracic Society. Comparison of four methods of smoking withdrawal in patients with smoking related smoking withdrawal in patien
diseases. BMF 1983;286:595-7.

19 Burt A, Illingworth D, Shaw T, et al. Stopping smoking after myocardial infarction. Lancet 1974;i:304-6.

20 Colby SM, Monti PM, Barnett NP, et al. Brief motivational interviewing in a hospital setting for adolescent smoking: a interviewing in a hospital setting for adolescent smoking:
preliminary study. $\mathcal{F}$ Consult Clin Psychol 1998;66:574-8.
21 Dale LC, Hurt RD, Offord KP, et al. High-dose nicotine patch therapy: percentage of replacement and smoking cessation. $7 A M A$ : 1995;274:1353-8.

22 Gritz ER, Carr CR, Rapkin D, et al. Predictors of long-term smoking cessation in head and neck cancer patients. Cancer Epidemiol Biomarkers Prev 1993;2:261-70.

23 Johnson JL, Budz B, Mackay M, et al. Evaluation of a nursedelivered smoking cessation intervention for hospitalized patients with cardiac disease. Heart Lung 1999;28:55-64.

24 Meenan RT, Stevens VJ, Hornbrook MC, et al. Costeffectiveness of a hospital-based smoking cessation intervention. Med Care 1998;36:670-8.

25 Schmitz JM, Spiga R, Rhoades HM, et al. Smoking cessation in women with cardiac risk: a comparative study of two theoretically based therapies. Nicotine Tobacco Res 1999;1: $87-94$.

26 Strecher VJ, Becker MH, Kirscht JP, et al. Evaluation of a minimal-contact smoking cessation program in a health
care setting. Patient Educ Counselling 1985;7:395-407.

27 Wewers ME, Bowen JM, Stanislaw AE, et al. A nursedelivered smoking cessation intervention among hospitalized postoperative patients-influence of a smoking-related diagnosis: a pilot study. Heart Lung 1994;23:151-6.

28 Stevens VJ, Glasgow RE, Hollis JF, et al. A smokingcessation intervention for hospital patients. Med Care 1993; 31:65-72.

29 Stevens VJ, Glasgow RE, Hollis JF, et al. Implementation and effectiveness of a brief smoking-cessation intervention for hospital patients. Med Care 2000;38:451-9.

30 Rigotti NA, Arnsten JH, McKool KM, et al. Efficacy of a smoking cessation program for hospital patients. Arch Intern Med 1997;157:2653-60.

31 Silagy C. Physician advice for smoking cessation. In: Cochrane Library, Issue 4, 2000. Oxford: Update Software.

32 Sciamanna CN, Stillman FA, Hoch JS, et al. Opportunities for improving inpatient smoking cessation programs: a community hospital experience. Prev Med 2000;30:496503.

33 Silagy C, Mant D, Fowler G, et al. Nicotine replacement therapy for smoking cessation. In: Cochrane Library, Issue 3, 2000. Oxford: Update Software.

34 Hughes JR, Stead LF, Lancaster T. Antidepressants for smoking cessation. In: Cochrane Library, Issue 4, 2000. Oxford: Update Software. 